\title{
Optimal management of nail disease in patients with psoriasis
}

\author{
This article was published in the following Dove Press journal: \\ Psoriasis: Targets and Therapy \\ 9 January 2015 \\ Number of times this article has been viewed
}

\section{Bianca Maria Piraccini Michela Starace \\ Division of Dermatology, Department of Experimental, Diagnostic and Specialty Medicine, University of Bologna, Bologna, Italy}

Abstract: Psoriasis is a common skin disease, with nail involvement in approximately $80 \%$ of patients. Nail psoriasis is often associated with psoriatic arthropathy. Involvement of the nails does not always have relationship with the type, gravity, extension, or duration of skin psoriasis. Nail psoriasis can occur at any age and all parts of the nails and the surrounding structures can be affected. Two clinical patterns of nail manifestations have been seen due to psoriasis: nail matrix involvement or nail bed involvement. In the first case, irregular and deep pitting, red spots of the lunula, crumbling, and leukonychia are seen; in the second case, salmon patches, onycholysis with erythematous border, subungual hyperkeratosis, and splinter hemorrhages are observed. These clinical features are more visible in fingernails than in toenails, where nail abnormalities are not diagnostic and are usually clinically indistinguishable from other conditions, especially onychomycosis. Nail psoriasis causes, above all, psychosocial and aesthetic problems, but many patients often complain about functional damage. Diagnosis of nail psoriasis is clinical and histopathology is necessary only in selected cases. Nail psoriasis has an unpredictable course but, in most cases, the disease is chronic and complete remissions are uncommon. Sun exposure does not usually improve and may even worsen nail psoriasis. There are no curative treatments. Treatment of nail psoriasis includes different types of medications, from topical therapy to systemic therapy, according to the severity and extension of the disease. Moreover, we should not underestimate the use of biological agents and new therapy with lasers or iontophoresis. This review offers an investigation of the different treatment options for nail psoriasis and the optimal management of nail disease in patients with psoriasis.

Keywords: biologics, nail psoriasis, topical therapy, systemic therapy

\section{Introduction}

Psoriasis is a chronic and recurrent inflammatory skin disease with nail involvement observed in approximately $80 \%$ of patients. Nail psoriasis may also occur as the only manifestation of the disease. ${ }^{1,2}$ Nail psoriasis can appear at any age and all nails can be affected. The nail matrix or nail bed can be involved in the disease. Nail psoriasis causes, above all, psychosocial and aesthetic problems, leading to significant repercussions in the quality of life, but many patients often also complain about functional damage. ${ }^{3}$ Cosmetic and functional damage, referred by $93 \%$ of patients with nail psoriasis, is sometimes so extensive that the patients tend to hide their hands and/or feet or shy away from social and business activities. ${ }^{4}$

The treatment of nail psoriasis involves different types of medications, from topical therapy to systemic therapy, according to the severity and extension of the disease.
Correspondence: Bianca Maria Piraccini Division of Dermatology, Department of Experimental, Diagnostic and Specialty Medicine, University of Bologna, Via Massarenti I, 40I38 Bologna, Italy Tel +390516364867

Fax +390516364867

Email biancamaria.piraccini@unibo.it (c) (i) (5) 2015 Piraccini and Starace. This work is published by Dove Medical Press Limited, and licensed under Creative Commons Attribution - Non Commercial (unported, v3.0) BY LC License. The full terms of the License are available at http://creativecommons.org/licenses//by-nd/3.0/. Non-commercial uses of the work are permitted without any further permission for the license are administered by Dove Medical Press Limited. Information on how to request permission may be found at: http://www.dovepress.com/permissions.php 
A standardized therapy does not exist, and the therapeutic choice is based on clinical manifestations and symptoms of the patient. Once the diagnosis has been defined, the severity of the disease should be assessed with a validated score, to decide the best treatment.

\section{Clinical manifestations}

Clinical manifestations of nail psoriasis depend on the nail site that is affected: the nail matrix, the nail bed, the proximal nail fold, or the hyponychium. ${ }^{5}$ It is possible to observe an isolated involvement of a few fingers or lesions of all nails on both hands and feet.

The severity of nail psoriasis depends largely on the nail part affected by the inflammatory reaction. The most severe changes are observed when the disease affects the nail matrix, a germinative epithelium that produces the nail plate. When the matrix is affected, psoriasis signs are pitting, leukonychia, lunula red spots, and nail plate crumbling (Figure 1). The nail bed is responsible for the adhesion of the nail plate; it is richly vascularized by capillaries oriented longitudinally in parallel ridges. Alterations of the nail bed due to nail psoriasis include onycholysis, subungual hyperkeratosis, oil drop or salmon spots, dyschromias, and splinter hemorrhages (Figure 2).

The most common sign of nail psoriasis is pitting, which is a focal defect of keratinization of the proximal matrix, with persistence of groups of nucleated and incompletely keratinized (parakeratotic) cells within the upper layers in the dorsal nail plate. These clusters poorly adhere to each other and are easily detachable, leaving pits on the surface of the nail plate. The term pitting describes the presence of small depressions on the nail plate surface ${ }^{6}$ (Figure 3 ). Psoriatic pits are usually large and deep, with irregular and uneven

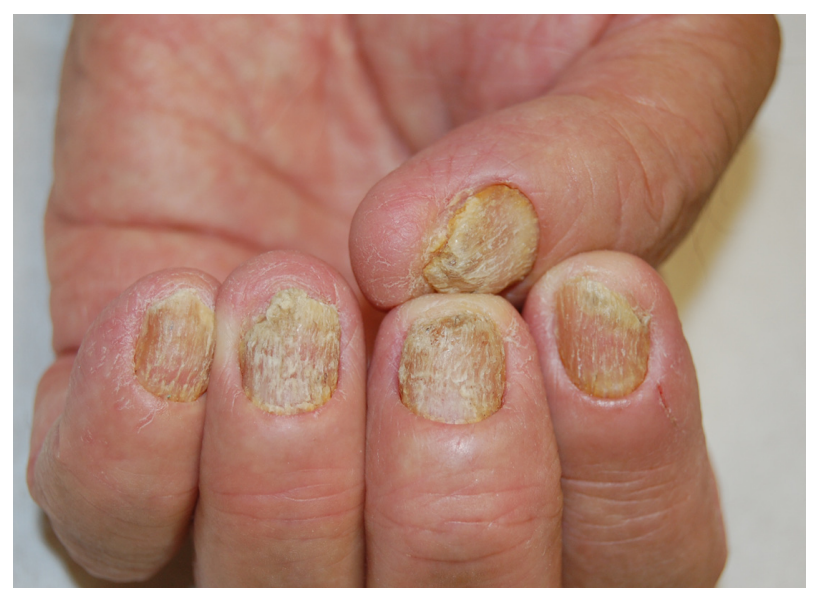

Figure I Nail matrix psoriasis of fingernails.

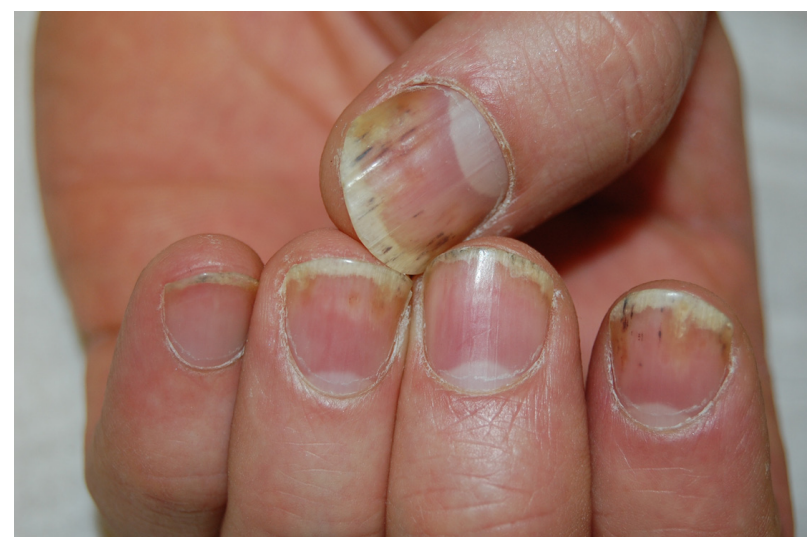

Figure 2 Nail bed psoriasis of fingernails.

shape and distribution; they may be covered by whitish, easily detachable scales. The fingernails are more often affected than the toenails. Pits may be the only manifestation of nail psoriasis or they may be associated with other signs.

Another possible sign of nail matrix psoriasis is the presence of red spots in the lunula (mottled lunula), a nonspecific sign that can also be due to nail lichen planus and eczema.

Severe nail matrix involvement induces nail plate crumbling, with a fragile and grossly deformed nail plate.

Onycholysis surrounded by an erythematous border is typical of nail bed psoriasis. Onycholysis is the detachment of the nail plate from the nail bed. In psoriasis, the onycholytic area is typically separated from the normal nail plate by an erythematous border ${ }^{6}$ (Figure 4).

Subungual hyperkeratosis describes the accumulation of scales under the distal portion of the nail plate, with nail thickening and uplifting. It most frequently involves the toenails. Splinter hemorrhages appear as longitudinal linear red-brown areas of hemorrhage, often seen in fingernails and located in the distal portion of the nail plate. They are a consequence of

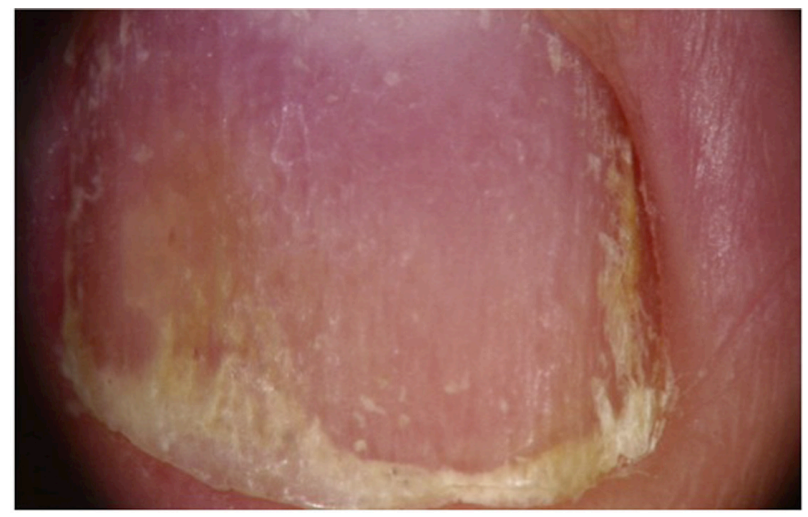

Figure 3 Enhanced visualization of pitting with dermoscopy (original magnification: $\times 20)$. 


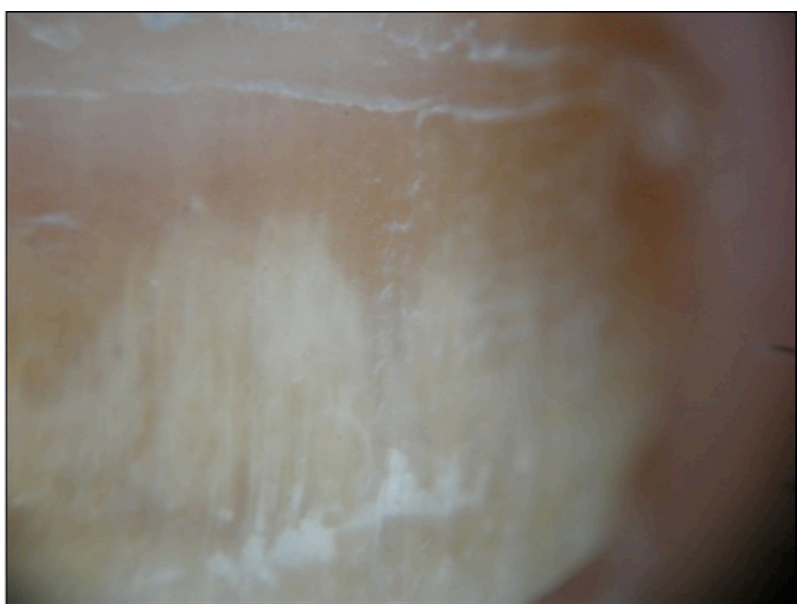

Figure 4 Enhanced visualization of onycholysis surrounded by an erythematous border with dermoscopy (original magnification: $\times 20$ ).

psoriatic involvement of the nail bed capillary vessels that run in a longitudinal direction along the nail bed dermal ridges. They are not specific of the disease.

Dyschromias of the nail are also frequent in nail psoriasis and the most typical signs are the 'oil drop sign' or salmon patches, which appear as irregular areas of yelloworange discoloration easily visible through the transparent nail plate. ${ }^{7}$

\section{Diagnosis}

The diagnosis of nail psoriasis is usually based on clinical manifestations. As the clinical features of nail psoriasis are not exclusive for the disease, clinical differentiation between other conditions, such as onychomycosis, can be difficult. The nail discoloration and subungual hyperkeratosis observed in nail psoriasis may also be present in onychomycosis. The use of dermoscopy, a noninvasive tool, can be useful in differential diagnosis: in nail psoriasis, it shows onycholysis with erythematous border (Figure 4), represented by a reddishorange stain surrounding the onycholytic area; whereas in onychomycosis, it shows a jagged proximal edge with spikes of onycholysis and longitudinal striae. ${ }^{8,9}$ Onychomycosis and psoriasis may also be present in the same nail. ${ }^{10}$ Another dermoscopic sign of nail psoriasis is the presence of vascular abnormalities in the hyponichium, where the capillaries are visible, dilated, tortuous, elongated, and irregularly distributed (Figure 5). The capillary density is correlated with the severity of the nail condition and improves with response to therapy. ${ }^{11}$ Periungual capillaroscopy may be a useful diagnostic tool for detecting nail psoriasis, where it shows a decreased capillary density in the periungual area and the presence of coiled capillary loops. ${ }^{12}$

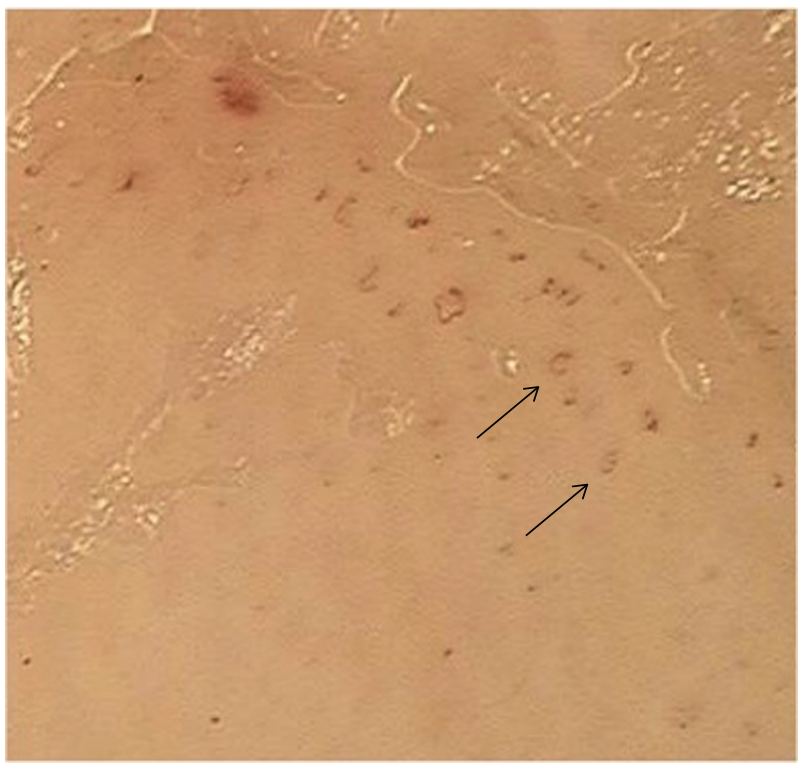

Figure 5 Dilated tortuous vessels of hyponychium with dermoscopy (original magnification: $\times 40$ ).

Note: The arrows indicate the tortuous vessels.

Nail biopsy maybe necessary in selected cases. Matrix or nail bed biopsy is performed based on the clinical manifestation of nail psoriasis. Hanno et $\mathrm{al}^{13}$ identified some criteria for pathological diagnosis of nail psoriasis: presence of neutrophils in nail bed epithelium as the major criterion; and hyperkeratosis with parakeratosis, exudates on corneal epithelium, focal hypogranulosis, and psoriasiform hyperplasia as minor criteria. It is important to perform periodic acidSchiff staining for differential diagnosis between psoriasis and onychomycosis. ${ }^{14}$

Recently, new diagnostic techniques have been suggested. Ultrasonography of the psoriatic nails shows hyperechoic parts or loss of definition of the nail plates, which can involve only the ventral part or the whole nail. The nail bed is thickened and shows an increased blood flow with power Doppler technique ${ }^{15}$ Optical coherence tomography provides images of tissue pathology in situ. In nail psoriasis, this technique shows a grossly irregular ventral nail plate irregularly fused with the underlying epidermis. ${ }^{16}$ Another new technique is confocal laser scanning microscopy, which can visualize, in a noninvasive manner, cell structures of the skin up to a depth of $300 \mu \mathrm{m}$ in vivo. This tool allows one to measure in a better way the thickness and the different layers of nail plate and gives better information on the microscopic structures than ultrasonography and optical coherence tomography. ${ }^{17}$

The most difficult differential diagnosis of nail psoriasis is onychomycosis, which may produce nail changes very similar to nail bed psoriasis. Another important disease to distinguish is nail lichen planus, which can be distinguished by absence of 
pitting and presence of longitudinal fissuring and, sometimes, dorsal pterygium. Pits are not exclusive to psoriasis; they can also be seen in alopecia areata and eczema. ${ }^{2}$ Nail pitting of alopecia areata is usually differentiated from that of nail psoriasis by the regular pattern of the depressions, which are superficial and geometrically distributed.

\section{Assessment of psoriatic nail disease}

There are many different scores specifically developed to assess the severity and to evaluate the courses of both disease and therapy in nail psoriasis. The only validated method to diagnose nail psoriasis is the NAPSI (Nail Psoriasis Severity Index), which is the most commonly used worldwide.

The NAPSI divides the affected nail into four quadrants, investigating each part for the presence of typical lesions. It exams both nail matrix (pitting, leukonychia, red spots of the lunula, and crumbling) and bed (oil drop patches, onycholysis, hyperkeratosis, and splinter hemorrhage). A value ranging from 0 to 8 is added for each nail affected; so the maximum score for each hand or foot is 0-160. The higher the NAPSI score, the worse is the nail involvement. ${ }^{18}$

Nail psoriasis causes both physical and psychological discomfort, and this is not included in the NAPSI score. In 2010, Ortonne et al $^{19}$ therefore devised the NPQ10 (Nail Psoriasis Quality of Life Scale) to evaluate the impact of nail psoriasis on quality of life. This questionnaire is a valid and reproducible method based on ten specific questions that investigate the impact of nail psoriasis on life. The questionnaire was administered to 1,309 patients and the results showed that nail psoriasis is evaluated as unsightly in $87 \%$ of patients and painful in $59 \%$ of patients. ${ }^{19}$ Klaassen et $\mathrm{al}^{20}$ showed that nail psoriasis can have devastating effects on quality of life, especially in female patients, in patients with associated psoriatic arthritis, and more particularly, in those with severe nail psoriasis. The NPQ10 highlighted the daily restrictions of patients with nail psoriasis, especially in patients with major chronic diseases such as cardiovascular disease, diabetes mellitus, and depression. Using this questionnaire was useful to individualize treatment strategies for each patient. ${ }^{20}$

The newest and internationally validated method of measuring the severity of nail psoriasis is the NAPPA (Nail Assessment in Psoriasis and Psoriatic Arthritis) score. This score evaluates both the quality of life and the therapeutic course of the disease. ${ }^{21}$ The NAPPA has been used in clinical studies as well as in routine care.

\section{Treatment of nail psoriasis}

The choice of treatment for nail psoriasis is a dilemma to the dermatologist for various reasons. To ensure that each patient receives the right treatment, it is necessary to evaluate many factors, such as the severity of the nail condition, the extension of the clinical manifestations on the skin, the joint involvement, and the previous response to therapeutic treatment. Often, patients have already been visited by other specialists without attaining good results and the impact on quality of life is very high. Sometimes, treatment failure occurs for various reasons that do not depend on the patient or dermatologist.

The nail has an anatomical structure that does not allow the absorption of topical medications at the level of the nail plate and this is amplified in nail psoriasis due to the presence of subungual hyperkeratosis. Nail psoriasis rarely responds to topical treatment and is often aggravated by exposure to the sun. The degree of penetration of topical medications is crucial. Furthermore, nail growth is very slow and therefore requires long-term treatment that may decrease patient compliance and increase the risk of drug toxicity. For this reason, the use of systemic drugs is recommended in cases of skin or joint involvement or in cases of severe nail psoriasis with poor therapeutic response to topical drugs.

Unfortunately, there is no standardized treatment regimen for the treatment of nail psoriasis and the choice of therapy depends on several factors, including the patient's history and clinical manifestations such as sex, age, severity of nail psoriasis, the disease's course over time, concomitant diseases and medications, and the benefits and risks of treatment.

Treatment options for nail psoriasis are summarized in Table 1.

Before starting treatment for nail psoriasis, it is necessary to consider various factors that are often underestimated. First of all, we must teach patients to keep nails short and cut off the onycholytic area so that topical therapies penetrate more and the exogenous material does not accumulate. Trauma should be avoided, so that the clinical manifestations do not deteriorate due to the Koebner phenomenon. For example, removal of the subungual material with sharp objects worsens the onycholysis.

\section{Topical therapies}

Topical treatments are often the first choice, but their effectiveness is limited to nail bed psoriasis; they are not effective on nail matrix psoriasis because they do not penetrate the nail plate and proximal nail fold. Topical treatments are formulated using different vehicles, including lacquer, creams, ointments, and tinctures, and may be applied 
Table I Treatment options for nail psoriasis

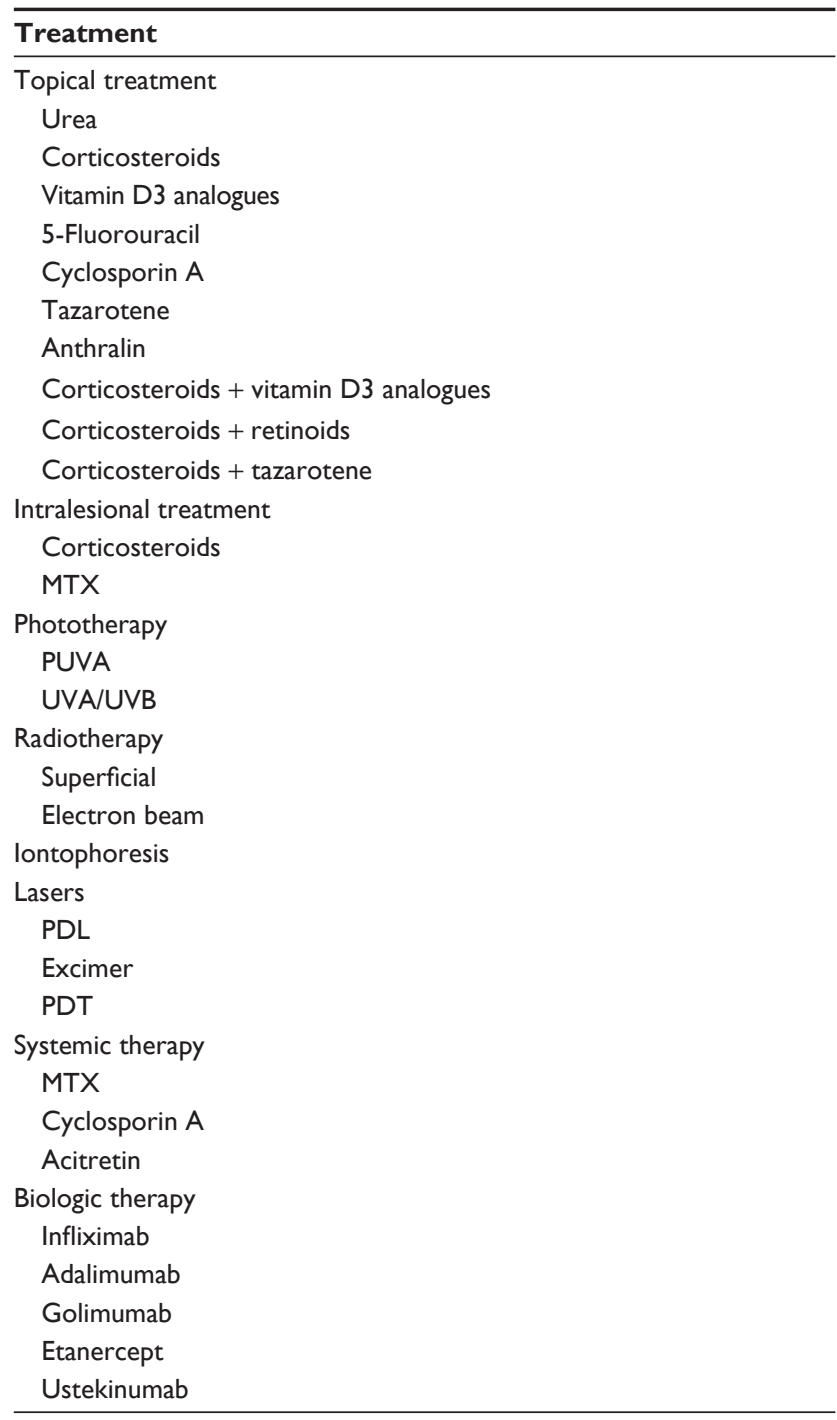

Abbreviations: MTX, methotrexate; PUVA, psoralen with UVA; UVA, ultraviolet A; UVB, ultraviolet B; PDL, pulsed-dye laser; PVT, photodynamic therapy.

on the nail plate or massaged into the nail bed and hyponychium or utilized under occlusion in order to increase their effect. Corticosteroids and vitamin D3 analogues are the first choice of treatment for nail bed psoriasis. Other local therapies used in nail psoriasis are 5-fluorouracil, cyclosporin A tazarotene, anthralin, and urea. Topical therapy is effective in nail bed psoriasis for the remedial action on onycholysis and subungual hyperkeratosis.

\section{Corticosteroids}

Potent or very potent corticosteroids are the most effective drugs in nail psoriasis, the most common being clobetasol propionate $(0.05 \%)^{22}$ and betamethasone dipropionate $(0.05 \%) .{ }^{23}$ They can be used once or twice daily. Recently, a pilot study ${ }^{24}$ of 15 patients published in 2012 compared the efficacy of clobetasol in nail lacquer at concentrations of $0.5 \%, 1.0 \%$, and $8.0 \%$. The formulation containing $8 \%$ clobetasol-17-propionate in colorless nail lacquer showed good clinical results and proved cosmetically highly acceptable for the treatment of nail bed and matrix psoriasis without joint involvement. ${ }^{24}$ The side effects of corticosteroids, especially under occlusion and when applied for long periods, are skin atrophy and, rarely, if prolonged for more than a year, the atrophy of the underlying phalanx known as "disappearing digit", ${ }^{25,26}$ formation of striae and telangiectasia, tachyphylaxis, and systemic absorption. ${ }^{6}$

\section{Vitamin D3 analogues}

The efficacy of topical calcipotriol in nail psoriasis was compared with that of the high-potency corticosteroids. In an open study, 62 patients were treated with topical calcipotriol once daily 5 days a week, as well as with clobetasol propionate $(0.05 \%)$ cream twice a week, for a total of 6 months. The authors reported a reduction of hyperkeratosis by $35.2 \%$ in fingernails and $72.3 \%$ in the toenails. ${ }^{22}$ In a controlled double-blind study of 58 patients for a period of 6 months, the authors demonstrated that twice a day of calcipotriol ointment had a similar efficacy as betamethasone dipropionate $(0.05 \%)$ in treating subungual hyperkeratosis after 3-9 months. ${ }^{23}$ The use of calcipotriol is preferred to avoid the side effects such as atrophy caused by steroids, but vitamin D3 analogues may cause erythema, periungual irritation, and burning sensation at the site of application and should never be applied under occlusion. After stopping therapy, the patient's nail condition generally worsens again.

\section{Tazarotene}

Tazarotene is a topical retinoid with already proven effectiveness in onycholysis, discoloration, pitting, and hyperkeratosis. It is used in gel or cream form for a period of 12-24 weeks. In 2007, Rigopoulos et $\mathrm{al}^{27}$ demonstrated a similar efficacy of tazarotene $(0.1 \%)$ cream and clobetasol propionate $(0.05 \%)$ cream treatment in a double-blind study involving 46 patients for 12 weeks. ${ }^{27}$ The drug may cause local irritation.

Other topical treatments that have been shown to be effective against nail psoriasis include tacrolimus, 5-fluorouracil, cyclosporin A, and anthralin.

\section{Combination therapies}

The use of combined local therapies has been demonstrated to produce better results in nail psoriasis. A combination of corticosteroids and vitamin D3 analogues shows better efficacy both on the clinical manifestations and on the symptoms reported by patients in nail bed psoriasis. Moreover, the 
effectiveness of corticosteroids can be enhanced in combination with keratolytic creams, such as topical retinoids or vitamin D3 analogues, allowing greater penetration. Finally, the combination of tazarotene with corticosteroids may help in reducing local irritation and thus enhance its effectiveness.

\section{Intralesional therapies}

Intralesional therapy is the injection of small doses of the drug directly into or near the specific structure of the nail unit: nail bed or nail matrix. The drug most frequently used in nail psoriasis is triamcinolone acetonide with a dose ranging from $2.5 \mathrm{mg} / \mathrm{mL}$ to $10 \mathrm{mg} / \mathrm{mL}$. There are many methods to inject drugs with different types of needles or with Dermo-Jet syringes. Doses, concentrations, and frequency of injections have not yet been standardized. The most important side effect is pain at the injection site, but this may be reduced with the use of ice or ethyl chloride spray.

Another drug recently used in the treatment of nail psoriasis is intralesional methotrexate. It was used at a dose of $2.5 \mathrm{mg}$ in a single finger of a patient with severe nail psoriasis at the level of the proximal nail fold, once a week for 6 weeks. During the 4-month follow-up, there was evidence of an improvement in pitting and subungual hyperkeratosis, and the results were maintained after 2 years. The only side effect reported was pain, but this is still defined as tolerable. ${ }^{28}$

\section{Phototherapy, radiotherapy}

Phototherapy (ultraviolet B, ultraviolet A [UVA], or psoralen with UVA (PUVA)) is a therapeutic option widely used in skin psoriasis, but it has never found support in nail psoriasis because the rays have a low ability to penetrate the nail plate. Phototherapy requires multiple sessions each week and a therapeutic effect is evident only after a long period. Published studies are not recent and showed an improvement only at the level of the proximal nail fold with the use of PUVA therapy, while no effect was reported with UVB therapy. A recent study proved the ability of penetration of UV lights in normal human cadaveric fingernails and suggested that PUVA could be considered a possible treatment in nail psoriasis, ${ }^{29}$ although clinical studies are needed.

Radiation therapy in nail psoriasis has been described in a few recent studies. Superficial radiotherapy induced a significant reduction in nail thickness in a double-blind study, ${ }^{30,31}$ while, more recently, a prospective study of electron beam therapy showed improvement in 9 of 12 patients with nail psoriasis and without joint involvement after 8 weeks of therapy, but the results were lost after 12 months of follow-up. ${ }^{32}$ However, in addition to the side effects of radiation therapy, such as fibrosis, inflammation, and local pigmentation, there is also the potential risk of carcinogenesis.

\section{Laser therapies}

Most studies on lasers for nail psoriasis were performed with the pulsed-dye laser (PDL), which specifically targets blood vessels. The most frequently chosen wavelengths for PDL therapeutic use are $585 \mathrm{~nm}$ and $595 \mathrm{~nm}$, which can effectively reach the nail bed through the nail plate. Most recently, PDL has been investigated in nail psoriasis. Four recent studies used PDL for nail psoriasis. The first study compared the efficacy of photodynamic therapy (PDT) and PDL in the therapy of nail psoriasis and compared treatment responses of lesions of the nail matrix versus those of the nail bed in a comparative pilot study of 14 patients. Results showed a reduction in NAPSI score in both treatment groups for both types of nail psoriasis without statistical differences between PDT and PDL. ${ }^{33}$ The second study evaluated the effect of PDL in nail psoriasis and showed an improvement on onycholysis and subungual hyperkeratosis in five patients. ${ }^{34}$ The third study compared, in 20 patients, the efficacy and safety of different types of lasers to determine the optimal pulse duration of PDL in nail psoriasis in a randomized, double-blind, intrapatient left-to-right study and showed no significant difference between the groups. ${ }^{35}$ The last study evaluated the efficacy and safety of PDL with topical retinoid in nail psoriasis of 25 patients and showed a marked improvement in the experimental group versus the control group in a single-blind, intrapatient left-to-right controlled study. ${ }^{36}$ These studies suggest that PDL may be clinically useful in treating lesions caused by nail matrix and bed psoriasis, but more randomized controlled trials are still needed to standardize PDL in nail psoriasis. Recently, a new single left-to-right comparison study, in 42 patients, compared excimer laser versus PDL and showed that PDL has a good response for treating nail psoriasis, with minimal side effects, while excimer laser is conversely more effective for plaque psoriasis than is PDL. ${ }^{37}$

\section{Systemic therapies}

Systemic therapy is recommended in patients with skin psoriasis or joint involvement but not for those with only nail manifestations. On account of the possible systemic toxicity, the European Consensus recommends the use of systemic therapy only in cases of moderate-to-severe nail psoriasis or when topical therapy, intralesional therapy, or phototherapy has failed. 
Systemic therapy used in nail psoriasis comprises immunosuppressant drugs such as methotrexate, cyclosporin A, and retinoids.

Methotrexate is one of the most important immunosuppressive drugs used in inflammatory diseases. The use of methotrexate in nail psoriasis is less studied than its use in skin psoriasis, and its effectiveness is not significantly different from that of other drugs. Methotrexate at low doses ( $5 \mathrm{mg} /$ week) has been used in a patient with severe 20-nail psoriasis with success in both the fingernails and toenails, which were cured after 9 months and 13 months of therapy, respectively. ${ }^{38}$ The dosage of methotrexate varies from $5 \mathrm{mg} /$ week to $15 \mathrm{mg} /$ week, and the recommended dose should not exceed $22.5 \mathrm{mg} /$ week. The effectiveness is evident after about 4-6 weeks of therapy. A recent study showed a reduction from $36.8 \%$ to $43.3 \%$ after $24-52$ weeks of therapy and showed a better response in nail matrix psoriasis. ${ }^{39}$ Possible side effects include liver and kidney toxicity and bone marrow suppression.

Data on the use of cyclosporin A in nail psoriasis are sparse. In a small, uncontrolled study of 16 patients with nail psoriasis and without joint involvement, where cyclosporin A was administered at a dose of $3 \mathrm{mg} / \mathrm{kg}$ of body weight, 14 of 16 patients improved, including 10 showing significant improvement and two cured completely. ${ }^{40}$ Another comparative single-blind study of 54 patients compared the effectiveness of cyclosporin A alone with a combination of cyclosporin A at a dose of $3.5-4.5 \mathrm{mg} /$ $\mathrm{kg} /$ day and topical calcipotriol (50 $\mu \mathrm{g} / \mathrm{kg}$ twice daily) for 3 months. Results showed an improvement in nail psoriasis of $79 \%$ in the combination group and $48 \%$ in patients receiving cyclosporin A alone. Furthermore, the relapses after 6 months were less in the combined group. ${ }^{41}$ In a retrospective evaluation, cyclosporin A was found to improve the NAPSI score after 12,24 , and 48 weeks by $40 \%, 72 \%$, and $89 \%$, respectively. ${ }^{42}$

Acitretin is a derivative of vitamin A used successfully in the treatment of pustular psoriasis. Therapeutic response is observed after 4-8 weeks (Figure 6A and B). An open study involving 36 patients with moderate-tosevere nail psoriasis with low-dose acitretin $(0.2-0.3 \mathrm{mg} /$ $\mathrm{kg}$ /day) showed an improvement of the NAPSI score in $40.9 \%$ patients and a complete healing in $25 \%$ patients after 6 months of therapy. ${ }^{43}$ On the contrary, a Brazilian open study conducted on 20 patients did not show any improvement after 4 months of therapy. ${ }^{44}$ Side effects are dryness of skin and mucous membranes, hypertriglyceridemia, and hepatitis.

\section{Biologics}

New treatments for nail psoriasis include biological therapies, which have already been proven effective in skin psoriasis and show interesting results in nail psoriasis. Biologics showed a high efficacy in the treatment of nail psoriasis and a higher response when compared to classical therapy, improving both signs and symptoms of severe nail psoriasis. No biological agent showed greater efficacy compared with another agent.

The most studied drug is infliximab, a chimeric monoclonal antibody that blocks tumor necrosis factor (TNF)- $\alpha$. A phase III multicenter, double-blind, placebo-controlled study evaluated the safety and efficacy of long-term infliximab treatment in 378 patients with moderate-to-severe plaque psoriasis. The second end point of the study was an improvement of nail manifestations. The percentage of improvement in NAPSI was $28.9 \%$ after 12 weeks and $51 \%$ after 54 weeks of therapy. A complete cure was reported in $30 \%$ of patients. ${ }^{45}$

Another antagonist of the TNF- $\alpha$ receptor is adalimumab. van den Bosch et $\mathrm{al}^{46}$ showed an improvement in NAPSI score of
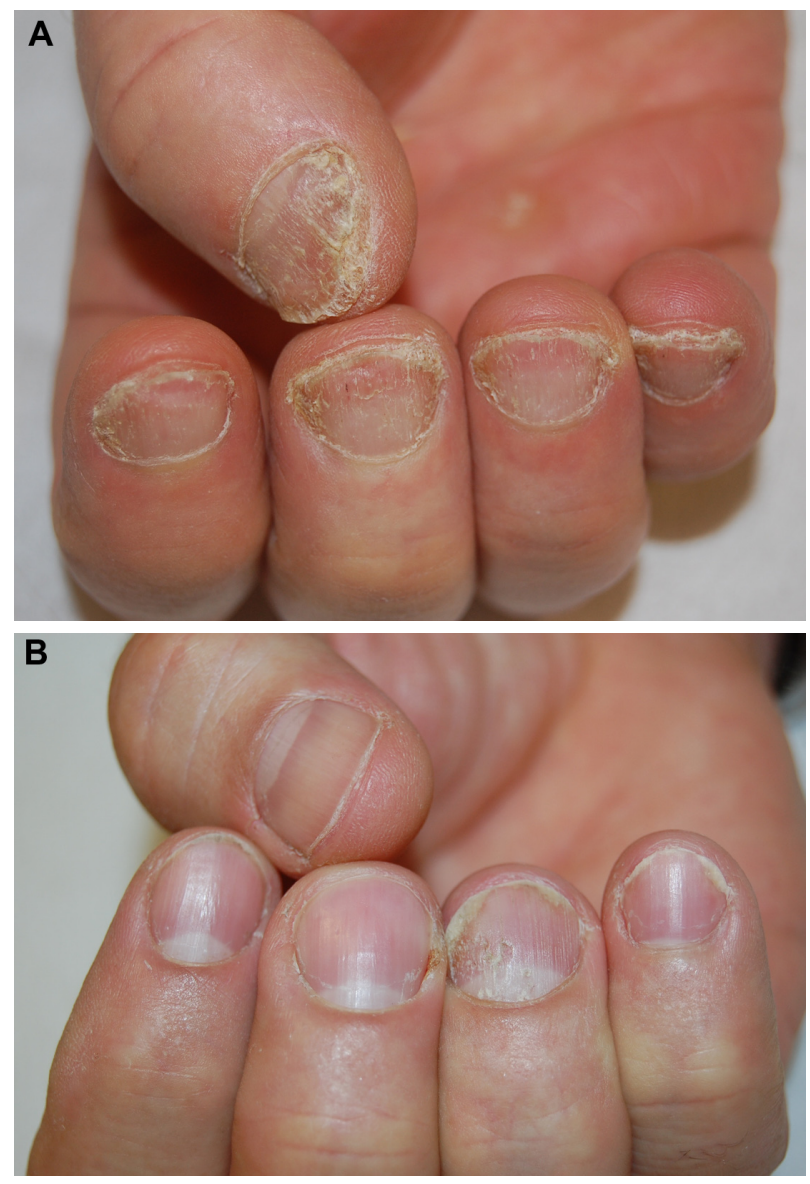

Figure 6 Nail psoriasis (A) before and (B) after treatment with acitretin. 
$65 \%$ after 20 weeks of therapy with adalimumab $40 \mathrm{mg} /$ week in an open-label uncontrolled study in 442 patients with psoriatic arthritis. Recently, a subanalysis of the effects on scalp and nails of adalimumab in severe psoriasis showed a $39.5 \%$ reduction of the NAPSI score after 16 weeks of treatment. ${ }^{47}$

In an open-label, randomized trial (CRYSTAL) of 711 patients, wherein $80 \%$ had nail involvement, etanercept showed a $51 \%$ reduction of NAPSI score after 54 weeks of therapy. ${ }^{48}$

Golimumab is a human monoclonal antibody. A study on arthropathic psoriasis showed a $33 \%$ reduction in the NAPSI score after 24 weeks. ${ }^{49}$

The biologic drug most recently used is ustekinumab, an interleukin-12/23 receptor inhibitor, which has shown good efficacy in nail psoriasis. ${ }^{50}$ Recently, the efficacy of ustekinumab in nail psoriasis was evaluated in an open-label study (PHOENIX 1), in 766 patients with moderate-to-severe psoriasis, where $71 \%$ presented nail disease. At week 24 of therapy, the rate of improvement in NAPSI score was $46.5 \%$. Ustekinumab showed excellent results in nail psoriasis and the improvement continued until after 1 year of treatment with the maintenance dose..$^{51}$

The debate is still open as to whether biologics, with their well-known side effects, are indicated for use in isolated nail psoriasis, where skin and joints have no symptoms.

\section{Conclusion}

Despite the pain and the functional and aesthetic discomfort, nail psoriasis is still a poorly studied disease. The evaluation of nail involvement is important for assessing the severity of psoriasis as it is an index of possible joint involvement, which may require a more substantial treatment. In cases of nail psoriasis, the clinical manifestations should first be observed in order to make a correct diagnosis and then to establish an appropriate treatment. Initially, local therapy may be useful, but in cases of cutaneous or joint involvement, systemic therapy is necessary with conventional or biological agents.

A 2013 Cochrane report on interventions in nail psoriasis assessed evidence for the efficacy and safety of treatments for the disease. They included 18 studies involving 1,226 patients and concluded that infliximab, golimumab, superficial radiotherapy, and electron beam brought about significant nail improvement compared to other treatments. However, the report also comments: "it was not possible to pool and compare the results because the studies were all so different". 52

In conclusion, psoriasis is a chronic and relapsing disease that needs further investigation to choose the best treatment for each individual patient.

\section{Disclosure}

The authors report no conflicts of interest in this work.

\section{References}

1. Cassell S, Kavanaugh AF. Therapies for psoriatic nail disease. A systematic review. J Rheumatol. 2006;33(7):1452-1456.

2. Tan ES, Chong WS, Tey HL. Nail psoriasis: a review. Am J Clin Dermatol. 2012;13:375-388.

3. Baran R. The Burden of nail psoriasis: an introduction. Dermatology. 2010;221(Suppl 1):1-5.

4. de Jong EM, Seegers BA, Gulinck MK, Boezeman JB, van de Kerkhof PC. Psoriasis of the nails associated with disability in a large number of patients: results of a recent interview with 1728 patients. Dermatology. 1996;193:300-303.

5. de Berker D. Nail anatomy. Clin Dermatol. 2013;31:509-515.

6. Jiaravuthisan MM, Sasseville D, Vender RB, Murphy F, Muhn CY. Psoriasis of the nail: anatomy, pathology, clinical presentation, and a review of the literature on therapy. J Am Acad Dermatol. 2007;57(1):1-27.

7. Edwards F, de Berker D. Nail psoriasis: clinical presentations and best practice recommendations. Drugs. 2009;69(17):2351-2361.

8. Farias DC, Tosti A, Chiacchio ND, Hirata SH. Dermoscopy in nail psoriasis. An Bras Dermatol. 2010;85:101-103.

9. Piraccini BM, Balestri R, Starace M, Rech G. Nail digital dermoscopy (onychoscopy) in the diagnosis of onychomycosis. J Eur Acad Dermatol Venereol. 2013;27:509-513.

10. Klaassen KMG, Dulak MG, van de Kerhof PCM, Pasch MC. The prevalence of onychomycosis in psoriatic patients: a systematic review. J Eur Acad Dermatol Venereol. 2014;28:533-541.

11. Iorizzo M, Dahdah M, Vincenzi C, et al. Videodermoscopy of the hyponychium in nail bed psoriasis. $J$ Am Acad Dermatol. 2008;58: 714-715.

12. Riberio CF, Siqueira EB, Holler AP, et al. Periungual capillaroscopy in psoriasis. An Bras Dermatol. 2012;87:550-553.

13. Hanno R, Mathes BM, Krull EA. Longitudinal nail biopsy in evaluation of acquired nail dystrophy. J Am Acad Dermatol. 1986;14:803-809.

14. Grover C, Reddy BS, Uma Chaturvedi K. Diagnosis of nail psoriasis: importance of biopsy and histopathology. Br J Dermatol. 2005;153: $1153-1158$.

15. Gutierrez M, Wortsman X, Filippucci E, De Angelis R, Filosa G, Grassi W. High-frequency sonography in the evaluation of psoriasis: nail and skin involvement. J Ultrasound Med. 2009;28:1569-1574.

16. Aydin SZ, Ash Z, Del Galdo F, et al. Optical coherence tomography: a new tool to assess nail disease in psoriasis? Dermatology. 2011;222: 311-313.

17. Sattler E, Kaestle R, Rothmund G, Welzel J. Confocal laser scanning microscopy, optical coherence tomography and transonychial water loss for in vivo investigation of nails. Br J Dermatol. 2012;166:740-746.

18. Rich P, Scher RK. Nail Psoriasis severity index: a useful tool for evaluation of nail psoriasis. J Am Acad Dermatol. 2003;49:206-212.

19. Ortonne JP, Baran R, Corvest M, Schmitt C, Voisard JJ, Taieb C. Development and validation of nail psoriasis quality of life scale (NPQ10). J Eur Acad Dermatol Venereol. 2010;24:22-27.

20. Klaassen KMG, van de Kerkhof PCM, Pasch MC. Nail psoriasis, the unknown burden of disease. J Eur Acad Dermatol Venereol. 2014;28(12):1690-1695.

21. Augustin M, Blome C, Costanzo A, et al. For the NAPPA task force group: conception, development and validation of NAPPA, a novel questionnaire for the assessment of patient-reported outcomes in nail psoriasis. Br J Dermatol. 2014;70:591-598.

22. Rigopoulos D, Ioannides D, Prastitis N, et al. Nail psoriasis: a combined treatment using calcipotriol cream and clobetasol propionate cream. Acta Derm Venereol. 2001;82:140.

23. Tosti A, Piraccini BM, Cameli N, et al. Calcipotriol ointment in nail psoriasis: a controlled double-blind comparison with betamethasone dipropionate and salicylic acid. Br J Dermatol. 1998;139:655-659. 
24. Nakamura RC, Abreu Ld, Duque-Estrada B, Tamler C, Leverone AP. Comparison of nail lacquer clobetasol efficacy at $0.05 \%, 1 \%$ and $8 \%$ in nail psoriasis treatment: prospective, controlled and randomized pilot study. An Bras Dermatol. 2012;87(2):203-211.

25. Wolf R, Tur E, Brenner S. Corticosteroid-induced "disappearing digit". J Am Acad Dermatol. 1990;23:755-756.

26. Requena L, Zamora E, Martin L. Acroatrophy secondary to longstanding applications of topical steroids. Arch Dermatol. 1990;126: 1013-1014.

27. Rigopoulos D, Gregoriou S, Katsambas A. Treatment of psoriatic nails with tazarotene $0.1 \%$ versus clobetasol propionate $0.05 \%$ cream: a double-blind study. Acta Derm Venereol. 2007;87:167-169.

28. Saricaoglu H, Oz A, Turan H. Nail psoriasis successfully treated with intralesional methotrexate: case report. Dermatology. 2011;222:5-7.

29. Stern DK, Creasey AA, Quijije J, Lebwohl MG. UVA and UVB penetration of normal human cadaveric fingernail plate. Arch Dermatol. 2011;147(4):439-441.

30. Finnerty EF. Successful treatment of psoriasis of the nails. Cutis. 1979;23:43-44.

31. Yu RCH, King CM. A double-blind study of superficial radiotherapy in psoriatic nail dystrophy. Acta Derm Venereol. 1992;72:124-136.

32. Kwang TY, Nee TS, Seng KT. A therapeutic study of nail psoriasis using electron beams. Acta Derm Venereol. 1995;75:90.

33. Fernández-Guarino M, Harto A, Sánchez-Ronco M, García-Morales I, Jaén P. Pulsed dye laser vs photodynamic therapy in the treatment of refractory nail psoriasis: a comparative pilot study. J Eur Acad Dermatol Venereol. 2009;23(8):891-895.

34. Oram Y, Karincaoğlu Y, Koyuncu E, Kaharaman F. Pulsed dye laser in the treatment of nail psoriasis. Dermatol Surg. 2010;36:377-381.

35. Treewittayapoom C, Singvahanont P, Chanprapaph K, Haneke E. The effect of different pulse durations in the treatment of nail psoriasis with 595-nm pulsed dye laser: a randomized, double-blind, intrapatient left-to-right study. J Am Acad Dermatol. 2012;66:807-812.

36. Huang YC, Chou CL, Chiang YY. Efficacy of pulsed dye laser plus topical tazarotene versus topical tazarotene alone in psoriatic nail disease: a single-blind, intrapatient left-to-right controlled study. Lasers Surg Med. 2013;45:102-107.

37. Al-Mutairi N, Noor T, Al-Haddad A. Single blinded left-to-right comparison study of excimer laser versus pulsed dye laser for treatment of nail psoriasis. Dermatol Ther. 2014;4(2):197-205.

38. Lee JY. Severe 20-nail psoriasis successfully treated by low dose methotrexate. Dermatol Online J. 2009;15(11):8-12.

39. Gümüşel M, Özdemir M, Mevlitoğlu I, Bodur S. Evaluation of the efficacy of methotrexate and cyclosporine therapies on psoriatic nails: a one-blind, randomized study. J Eur Acad Dermatol Venereol. 2011;25(9):1080-1084.
40. Syuto T, Abe M, Ishibuchi H, et al. Successful treatment of psoriatic nail with low-dose cyclosporine administration. Eur J Dermatol. 2007;17(3):248-249.

41. Feliciani C, Zampetti A, Forleo P, et al. Nail psoriasis: combined therapy with systemic cyclosporine and topical calcipotriol. J Cutan Med Surg. 2004;8:122-125.

42. Sánchez-Regaña M, Sola-Ortigosa J,Alsina-Gibert M, Vidal-FernándezM, Umbert-Millet P. Nail psoriasis: a retrospective study on the effectiveness of systemic treatments (classical and biological therapy). J Eur Acad Dermatol Venereol. 2011;25(5):579-586.

43. Tosti A, Ricotti C, Romanelli P, Cameli N, Piraccini BM. Evaluation of the efficacy of acitretin therapy for nail psoriasis. Arch Dermatol. 2009;145(3):269-271.

44. Mukai MM, Poffo IF, Werner B, Brenner FM, Lima Filho JH. NAPSI utilization as an evaluation method of nail psoriasis in patients using acitretin. An Bras Dermatol. 2012;87(2):256-262.

45. Reich K, Nestle FO, Papp K, EXPRESS study investigators, et al. Infliximab induction and maintenance therapy for moderate-tosevere psoriasis: a phase III, multicenter, double-blind trial. Lancet 2005;366(9494):1367-1374.

46. van den Bosch F, Manger B, Goupille P, et al. Effectiveness of adalimumab in treating patients with active psoriatic arthritis and predictors of good clinical responses for arthritis, skin and nail lesions. Ann Rheum Dis. 2010;69(2):394-399.

47. Thaci D, Unnebrink K, Sundaram M, et al. Adalimumab for the treatment of moderate to severe psoriasis: subanalysis of effects on scalp and nails in the BELIEVE study. J Eur Acad Dermatol Venereol. In press.

48. Luger TA, Barker J, Lambert J, et al. Sustained improvement in joint pain and nail symptoms with etanercept therapy in patients with moderate-to-severe psoriasis. J Eur Acad Dermatol Venereol. 2009;23(8):896-904.

49. Kavanaugh A, McInnes I, Mease P, et al. Golimumab, a new human tumor necrosis factor alpha antibody, administered every four weeks as a subcutaneous injection in psoriatic arthritis. Arthritis Rheum. 2009;60 976-986.

50. Vitiello M, Tosti A, Abuchar A, et al. Ustekinumab for the treatment of nail psoriasis in healthy treated psoriatic patients. Int J Dermatol. 2013;52:358-362.

51. Rich P, Bourcier M, Sofen H, PHOENIX 1 investigators, et al. Ustekinumab improves nail disease in patients with moderate-to-severe psoriasis: results from PHOENIX 1. Br J Dermatol. 2014;170(2): 398-407.

52. de Vries AC, Bogaards NA, Hooft L, et al. Interventions for nail psoriasis. Cochrane Database Syst Rev. 2013;1:CD007633.
Psoriasis: Targets and Therapy

\section{Publish your work in this journal}

Psoriasis: Targets and Therapy is international, peer-reviewed, open access journal focusing on psoriasis, nail psoriasis, psoriatic arthritis and related conditions, identification of therapeutic targets and the optimal use of integrated treatment interventions to achieve improved outcomes and quality of life. The manuscript management system

\section{Dovepress}

is completely online and includes a very quick and fair peer-review system. Visit http://www.dovepress.com/testimonials.php to read real quotes from published authors. 